\title{
论 文
}

\section{储気球形压力容器中氚的渗透分析}

\author{
尹益辉 ${ }^{(1)}$, 刘远东 ${ }^{(1 *}$, 陈长安 ${ }^{(2)}$, 谭云 ${ }^{(1)}$ \\ (1) 中国工程物理研究院总体工程研究所, 绵阳 621900; \\ (2) 表面物理与化学国防科技重点实验室, 绵阳 621907 \\ *E-mail: liuyd@caep.ac.cn
}

收稿日期: 2016-01-31; 接受日期: 2016-06-08; 网络出版日期: 2016-09-22

中物院中子物理学重点实验室基金(批准号: 2014BB01)和中国工程物理研究院重点学科项目“计算固体力学”资助

\begin{abstract}
摘要随着时间延长, 储存在压力容器中的気大部分衰变产生氦-3, 极少部分通过容器内表面渗透到容器壁 材料中. 在容器壁中的気又一边扩散直至通过容器外表面渗透到容器外, 一边衰变产生氦-3 滞留在壁中. 渗透 到容器外部的気会污染环境, 危害人身健康与安全, 因此需要对其渗透量加以分析和防控. 针对容器外表面为 一般传质边界条件和容器内部気为范德华气体的情况, 同时考虑容器内部気的衰变与渗透和容器壁中気的衰 变、扩散与渗透, 建立了储凩容器中気向容器外渗透的解析理论模型, 导出了通过容器外表面的気渗透总量和衰 变后剩余量的理论公式. 通过解析计算给出了容器外気渗透总量和衰变后剩余量随时间的变化规律, 以及気渗 透总量随容器外表面传质系数和容器壁厚的变化规律. 与理论计算对应, 开展気储存实验与测试, 对储気容器 外部密封包装容器内的氞保护气取样, 采用正比计数器方法测量気浓度, 再换算得到了容器外気的瞬态量. 对 比分析了解析计算结果与实测结果, 表明了理论计算的合理性. 所得到的気渗透量随储存时间等参数的变化规 律对储衾容器的设计具有理论指导作用.
\end{abstract}

关键词球形压力容器, 気衰变, 渗透, 扩散

\section{1 引言}

気除了在军事上的应用外，在工农业、生物医 学、水文地质、科学研究中的应用也越来越广泛. 这 些应用使気的生产量、操作量和储存量变得越来越大. 氞是一种放射性气体, 其半衰期长达 $4501 \mathrm{~d}$. 虽然気 的 $\beta$ 能量很低, 外照射的危害很小, 但気进入人体可 与细胞中的 DNA 和 RNA 结合, 诱发染色体发生畸变, 特别是由 $3 \mathrm{H}$ 衰变为 $3 \mathrm{He}$ 时, 可能导致基因突变, 引
起不良遗传效应等内辐照危害 ${ }^{[1]}$. 因此, 认识和监测 涉気过程中気向周围环境的外泄规律, 控制気对环 境的污染及对人员的危害, 是具有现实意义的事情. 在涉気过程中, 気的临时和长期储存是一个重要环 节. 特别是気的长期储存, 不仅要关心気的渗透流失, 还要关心渗透気对环境的污染. 分析、认识储気容器 中氡的渗透规律, 反过来可以为改进储存容器与储 存方式, 以及为控制氞的流失和污染提供基础认识.

文献[2]指出, 对于高压气相気储存容器中氞的

引用格式: 尹益辉, 刘远东, 陈长安, 等. 储気球形压力容器中気的渗透分析. 中国科学: 技术科学, 2016, 46: 1071-1078

Yin Y H, Liu Y D, Chen C A, et al. Analysis on tritium permeation in spherical pressure vessel (in Chinese). Sci Sin Tech, 2016, 46: 1071-1078, doi: 10.1360/N092014-00364 
渗透, 一般来说扩散为速率限制过程, 因此, 根据 Fick's 定律, 容器壁外表面处気的扩散通量就为渗透 通量, 即在材料扩散系数和気浓度确定的情况下, 気 的渗透就可由 Fick's 定律直接计算得到. 以前, 针对 気渗透的研究, 大多是实验测试材料的気渗透率, 基 于实测数据了解材料的抗気渗透性能 ${ }^{[3 \sim 7]}$, 或是利用 实测数据计算容器或结构系统的気渗透量 ${ }^{[8]}$. 在后者 的理论计算中, 主要是采用稳态渗透模型, 以至忽略 了容器外表面处気的瞬时浓度梯度效应; 即使有文 献采用瞬态渗透理论计算, 但在気浓度求解中也引 入了诸如外边界上気浓度恒为零等假设条件 ${ }^{[9]}$. 有别 于这些情况, 文献 $[10,11]$ 针对気和氦分别为理想气 体或范德华气体、容器外表面分别为第一类传质边界 条件或第三类传质边界条件的情况, 建立了求解储 気容器壁中気和氦-3 浓度的解析理论模型, 导出了 各情况下容器壁中気和氦-3 浓度的理论公式, 为理 论计算瞬态気渗透提供了更加精确的前提. 本文在 文献[11]的基础上进一步推导気通过容器外表面的 渗透量及其衰变后剩余量公式, 并通过理论计算, 得 到了渗透量随有关变量的变化规律, 以及与实测和 稳态理论计算结果相比的差别.

\section{2 理论模型与计算公式}

\section{1 考虑気衰变与扩散时器壁中気的浓度}

文献[11]研究了储存高压气相丞的容器壁中氞 的瞬态扩散问题, 其考虑的初始条件为

$$
c_{\mathrm{T}}\left(r, r \neq R_{\mathrm{i}}, t=0\right)=0,
$$

式中 $c_{\mathrm{T}}$ 是器壁中固溶気浓度, 本文为体积浓度(原子 数 $\left./ \mathrm{m}^{3}\right), r$ 和 $t$ 分别是球坐标变量和时间变量, $R_{\mathrm{i}}$ 是 容器内径.

考虑的容器内表面边界条件为

$$
c_{\mathrm{T}}\left(r=R_{\mathrm{i}}, t\right)=S_{0 \mathrm{~T}} \sqrt{P_{\mathrm{T}}} \exp \left(-Q_{\mathrm{S}} / R T\right),
$$

式中 $S_{0 \mathrm{~T}}$ 是気在器壁材料中的溶解度常数; $P_{\mathrm{T}}$ 为容 器中气相氚引起的压强; $Q_{S}$ 是溶解热; $R$ 是普适气 体常数, 其值为 $8.31451 \mathrm{~J} \mathrm{~mol}^{-1} \mathrm{~K}^{-1} ; T$ 是绝对温度. (2)式即是 Sieverts 定律, 它表示容器内表面处気的浓 度与容器内部気分压的平方根成正比. 显然, (2)式与 第一类对流传质边界条件不同, 因为在第一类对流 传质边界条件中直接取容器表面気浓度与环境気浓 度相等.
考虑的容器外表面传质边界条件为

$$
-\left.D_{\mathrm{T}} \frac{\partial c_{\mathrm{T}}}{\partial r}\right|_{r=R_{\mathrm{o}}}=\eta\left(\left.c_{\mathrm{T}}\right|_{r=R_{\mathrm{o}}}-c_{\mathrm{Tf}}\right),
$$

式中 $D_{\mathrm{T}}$ 是扩散系数, $D_{\mathrm{T}}=D_{0 \mathrm{~T}} \exp \left(-Q_{\mathrm{D}} / R T\right), D_{0 \mathrm{~T}}$ 是 扩散常数, $Q_{\mathrm{D}}$ 是扩散激活能; $R_{\mathrm{o}}$ 是容器外径; $\eta$ 是 对流传质系数, 其单位为 $\mathrm{m} / \mathrm{s} ; c_{\mathrm{Tf}}$ 是与器壁外表面接 触的空气中的気浓度. (3)式是第三类对流传质边界 条件, 为一般情况的边界条件, 当 $\eta=\infty$ 或 $\eta=0$ 时, 它就分别退化为外表面気浓度恒定不变的第一类边 界条件和外表面気浓度梯度恒为 0 的第二类传质边 界条件, 而第一、第二类对流传质边界条件属于边界 对流传质的两种特殊情况, 也是两个极端情况.

基于上述初始和边界条件, 文献[11]考虑容器内 部気的衰变、渗透和容器壁中氞的扩散、衰变及衰变 氦-3 的滞留, 建立解析求解模型, 推导出了容器壁中 気的无量纲浓度为

$$
\begin{aligned}
\bar{c}_{\mathrm{T}}(\bar{r}, \bar{t})= & \frac{1}{\bar{r}} \sum_{n=1}^{\infty} A_{n}(\bar{t}) \mathrm{e}^{-\left(\bar{\lambda}+\xi_{n}^{2}\right) \bar{t}} \psi_{n}(\bar{r}) \\
& +\sqrt{\bar{P}_{\mathrm{T}}}\left(\bar{r}_{\mathrm{o}}-\bar{r}\right)+\frac{\sqrt{\bar{P}_{\mathrm{T}}}}{1+\bar{\eta}}\left(\bar{r}-\overline{r_{\mathrm{i}}}\right),
\end{aligned}
$$

其中 $\xi_{n}(n=1,2, \cdots, \infty)$ 是由特征方程

$$
\bar{r}_{\mathrm{o}} \bar{\eta} \sin \xi-\sin \xi+\bar{r}_{\mathrm{o}} \xi \cos \xi=0,
$$

确定的特征根, 而

$$
\begin{aligned}
A_{n}(\bar{t})= & {\left[\left(\frac{F_{2 n}}{F_{1 n}}-\frac{F_{3 n}}{F_{1 n}} \frac{1}{1+\bar{\eta}}\right) \frac{\bar{\lambda}}{2}-\frac{F_{4 n}}{F_{1 n}} \frac{2 \bar{\eta}}{1+\bar{\eta}}\right] } \\
& \times \frac{\beta_{1}}{\bar{\lambda} / 2+\xi_{n}^{2}}\left[\mathrm{e}^{\left(\bar{\lambda} / 2+\xi_{n}^{2}\right) \bar{t}}-1\right] \\
& -\left[\left(\frac{F_{2 n}}{F_{1 n}}-\frac{F_{3 n}}{F_{1 n}} \frac{1}{1+\bar{\eta}}\right) \bar{\lambda}+\frac{F_{4 n}}{F_{1 n}} \frac{2 \bar{\eta}}{1+\bar{\eta}}\right] \\
& \times \frac{\beta_{2}}{\xi_{n}^{2}-\bar{\lambda}}\left[\mathrm{e}^{\left(\xi_{n}^{2}-\bar{x}\right) \bar{t}}-1\right]+\frac{F_{2 n}}{F_{1 n}}-\frac{F_{3 n}}{F_{1 n}} \frac{1}{1+\bar{\eta}},
\end{aligned}
$$

$\psi_{n}(\bar{r})=\sin \xi_{n} \bar{r}-\operatorname{tg} \xi_{n} \bar{r}_{\mathrm{i}} \cos \xi_{n} \bar{r}$. 在 $A_{n}(\bar{t})$ 中,

$$
\begin{aligned}
& F_{1 n}=\int_{\bar{T}_{\bar{T}}}^{\bar{T}_{0}}\left[\psi_{n}(\bar{r})\right]^{2} \mathrm{~d} \bar{r}, \\
& F_{2 n}=\int_{\bar{T}_{\bar{T}}}^{\bar{T}_{0}} \bar{r}\left(\bar{r}-\bar{r}_{\mathrm{o}}\right) \psi_{n}(\bar{r}) \mathrm{d} \bar{r}, \\
& F_{3 n}=\int_{\bar{T}_{\bar{T}}}^{\bar{T}_{\bar{r}}} \bar{r}\left(\bar{r}-\overline{r_{\mathrm{i}}}\right) \psi_{n}(\bar{r}) \mathrm{d} \bar{r}, \\
& F_{4 n}=\int_{\bar{T}_{\overline{1}}}^{\bar{T}_{\bar{T}}} \psi_{n}(\bar{r}) \mathrm{d} \bar{r} .
\end{aligned}
$$

在这些式子中, 无量刚变量 $\bar{r}=r / h, \bar{r}_{\mathrm{i}}=R_{\mathrm{i}} / h$, $\bar{r}_{\mathrm{o}}=R_{\mathrm{o}} / h, \quad \bar{t}=t D_{\mathrm{T}} / h^{2}, \quad D_{\mathrm{T}}=D_{0 \mathrm{~T}} \exp \left(-Q_{\mathrm{D}} / R T\right), \quad \bar{c}_{\mathrm{T}}$ 
$=c_{\mathrm{T}} /\left(S_{\mathrm{T}} \sqrt{P_{\mathrm{T} 0}}\right), \quad S_{\mathrm{T}}=S_{0 \mathrm{~T}} \exp \left(-Q_{\mathrm{S}} / R T\right), \bar{P}_{\mathrm{T}}=P_{\mathrm{T}} / P_{\mathrm{T} 0}$, $\bar{\lambda}=\lambda h^{2} /\left[D_{0 \mathrm{~T}} \exp \left(-Q_{\mathrm{D}} / R T\right)\right]$ 和 $\bar{\eta}=\eta h /\left[D_{0 \mathrm{~T}} \exp \left(-Q_{\mathrm{D}}\right.\right.$ $/ R T)$ ]; 而 $h$ 是容器壁厚; $P_{\mathrm{T} 0}$ 是初始时刻容器中的 気压强; $\lambda$ 是気的衰变常数, $\lambda=\ln 2 / \tau_{1 / 2}$, 而 $\tau_{1 / 2}$ 是 気的半衰期, 其值为 $4501 \mathrm{~d} ; S_{\mathrm{T}}$ 是気在器壁材料中 的溶解度.

\section{2 通过容器外表面的氞渗透总量与衰变后剩余量}

容器内部储存的高压气相気向容器外的渗透, 涉及内、外表面上気分子的吸附/解吸和离解/复合、以 及表面与体内之间交换氢原子和氢在晶格内扩散等 4 个分过程. 这些分过程中速度最慢的就成为限制渗 透速率的过程. 一般情况下吸附/解吸和表面与体内 之间交换氢原子两种分过程的速率很大, 因此限制 渗透速率的是离解/复合和氢在晶格内扩散两个分过 程. 而对于储存氢或其同位素的钢容器, 一般来说扩 散为渗透速率的限制过程 [2]. 因此本文研究的储気 高压容器的渗透问题实际上就是扩散过程控制渗透 的情况. 该情况下, 边界条件(3)式中参数 $\bar{\eta}$ 对渗透的 影响是且仅是通过对扩散(即気浓度)的影响而实现 的，而与表面吸附/离解、解吸/复合等表面过程无关. 这样, 由 Fick's 第一定律, 容器壁中気的扩散通量为

$$
J=-D_{\mathrm{T}} \frac{\partial c_{\mathrm{T}}}{\partial r} .
$$

再由(3)式, 在容器外表面处有

$$
J=-\left.D_{\mathrm{T}} \frac{\partial c_{\mathrm{T}}}{\partial r}\right|_{r=R_{\mathrm{o}}}=\eta\left(\left.c_{\mathrm{T}}\right|_{r=R_{\mathrm{o}}}-c_{\mathrm{Tf}}\right) .
$$

将(4)式还原成 $c_{\mathrm{T}}(r, t)$, 再代入(8)式, 并对整个 容器外表面积分, 得到気通过容器外表面的总渗透 速率为

$$
\begin{aligned}
\frac{\mathrm{d} Q_{\mathrm{p} 0}(t)}{\mathrm{d} t}= & 4 \pi D_{\mathrm{T}} S_{\mathrm{T}} \sqrt{P_{\mathrm{T} 0}} h \\
& \times\left\{\sum_{n=1}^{\infty} A_{n}(t) \mathrm{e}^{-\left(\lambda+\lambda \xi_{n}^{2} \bar{\lambda}\right) t} \frac{\sin \xi_{n}-\bar{r}_{\mathrm{o}} \xi_{n} \cos \xi_{n}}{\cos \xi_{n} \bar{r}_{\mathrm{i}}}\right. \\
& \left.+\frac{\bar{\eta} \bar{r}_{\mathrm{o}}^{2}}{1+\bar{\eta}} \sqrt{\bar{P}_{\mathrm{T}}(t)}\right\},
\end{aligned}
$$

其中 $Q_{\mathrm{p} 0}(t)$ 是任意时刻気的渗透量, $D_{\mathrm{T}} S_{\mathrm{T}}$ 是扩散系 数和溶解度之积, 称为渗透系数.

积分(9)式得到容器内部気渗透到容器外部的总 量为
$Q_{\mathrm{p} 0}(t)=4 \pi D_{\mathrm{T}} S_{0 \mathrm{~T}} \exp \left(-\Delta H_{\mathrm{T}} / R T\right) \sqrt{P_{\mathrm{T} 0}} h$

$$
\times\left\{\sum_{n=1}^{\infty} B_{1 n}(t) \frac{\sin \xi_{n}-\bar{r}_{0} \xi_{n} \cos \xi_{n}}{\cos \xi_{n} \bar{r}_{\mathrm{i}}}+\frac{\bar{\eta} \bar{r}_{0}^{2}}{1+\bar{\eta}} B_{2}(t)\right\},
$$

其中

$$
\begin{aligned}
B_{1 n}(t)= & {\left[\left(\frac{F_{2 n}}{F_{1 n}}-\frac{F_{3 n}}{F_{1 n}} \frac{1}{1+\bar{\eta}}\right) \frac{\bar{\lambda}}{2}-\frac{F_{4 n}}{F_{1 n}} \frac{2 \bar{\eta}}{1+\bar{\eta}}\right] \frac{\beta_{1}}{\bar{\lambda} / 2+\xi_{n}^{2}} } \\
& \times\left[\frac{2}{\lambda}\left(1-\mathrm{e}^{-\lambda t / 2}\right)+\frac{1}{\lambda+\lambda \xi_{n}^{2} / \bar{\lambda}}\left(\mathrm{e}^{-\left(\lambda+\lambda \xi_{n}^{2} / \bar{\lambda}\right) t}-1\right)\right] \\
& -\left[\left(\frac{F_{2 n}}{F_{1 n}}-\frac{F_{3 n}}{F_{1 n}} \frac{1}{1+\bar{\eta}}\right) \bar{\lambda}+\frac{F_{4 n}}{F_{1 n}} \frac{2 \bar{\eta}}{1+\bar{\eta}}\right] \frac{\beta_{2}}{\xi_{n}^{2}-\bar{\lambda}} \\
& \times\left[\frac{1}{2 \lambda}\left(1-\mathrm{e}^{-2 \lambda t}\right)+\frac{1}{\lambda+\lambda \xi_{n}^{2} / \bar{\lambda}}\left(\mathrm{e}^{-\left(\lambda+\lambda \xi_{n}^{2} \bar{\lambda}\right) t}-1\right)\right] \\
+ & \left(\frac{F_{2 n}}{F_{1 n}}-\frac{F_{3 n}}{F_{1 n}} \frac{1}{1+\bar{\eta}}\right) \frac{1}{\lambda+\lambda \xi_{n}^{2} / \bar{\lambda}}\left(1-\mathrm{e}^{-\left(\lambda+\lambda \xi_{n}^{2} \bar{n}\right) t}\right), \\
& B_{2}(t)=\frac{2 \beta_{1}}{\lambda}\left(1-\mathrm{e}^{-\lambda t / 2}\right)+\frac{\beta_{2}}{2 \lambda}\left(1-\mathrm{e}^{-2 \lambda t}\right),
\end{aligned}
$$

式中 $\beta_{1}=0.894790, \beta_{2}=0.111518$, 其具体确定方法 可见文献[11].

渗透到容器外部的気在不断累积的同时, 也衰 变成为氦-3. 容器外部渗透気衰变后剩余量 $Q_{\mathrm{p} 1}$ 满足 关系式

$$
\frac{\mathrm{d} Q_{\mathrm{p} 1}}{\mathrm{~d} t}=-\lambda Q_{\mathrm{p} 1}+\frac{\mathrm{d} Q_{\mathrm{p} 0}}{\mathrm{~d} t} .
$$

求解该式得到

$$
\begin{aligned}
Q_{\mathrm{p} 1}(t) & =4 \pi D_{\mathrm{T}} S_{0 \mathrm{~T}} \exp \left(-\Delta H_{\mathrm{T}} / R T\right) \sqrt{P_{\mathrm{T} 0}} h \\
& \times\left\{\sum_{n=1}^{\infty} B_{3 n}(t) \frac{\sin \xi_{n}-\bar{r}_{0} \xi_{n} \cos \xi_{n}}{\cos \xi_{n} \bar{r}_{\mathrm{i}}}+\frac{\bar{\eta} \bar{r}_{\mathrm{o}}^{2}}{(1+\bar{\eta})} B_{4}(t)\right\} \mathrm{e}^{-\lambda t},
\end{aligned}
$$

其中

$$
\begin{aligned}
B_{3 n}(t)= & {\left[\left(\frac{F_{2 n}}{F_{1 n}}-\frac{F_{3 n}}{F_{1 n}} \frac{1}{1+\bar{\eta}}\right) \frac{\bar{\lambda}}{2}-\frac{F_{4 n}}{F_{1 n}} \frac{2 \bar{\eta}}{1+\bar{\eta}}\right] \frac{\beta_{1}}{\bar{\lambda} / 2+\xi_{n}^{2}} } \\
& \times\left[\frac{2}{\lambda}\left(\mathrm{e}^{\lambda t / 2}-1\right)-\frac{\bar{\lambda}}{\lambda \xi_{n}^{2}}\left(1-\mathrm{e}^{-\left(\lambda \xi_{n}^{2} \bar{\lambda}\right) t}\right)\right] \\
& -\left[\left(\frac{F_{2 n}}{F_{1 n}}-\frac{F_{3 n}}{F_{1 n}} \frac{1}{1+\bar{\eta}}\right) \bar{\lambda}+\frac{F_{4 n}}{F_{1 n}} \frac{2 \bar{\eta}}{1+\bar{\eta}}\right] \frac{\beta_{2}}{\xi_{n}^{2}-\bar{\lambda}} \\
& \times\left[\frac{1}{\lambda}\left(1-\mathrm{e}^{-\lambda t}\right)-\frac{\bar{\lambda}}{\lambda \xi_{n}^{2}}\left(1-\mathrm{e}^{-\left(\lambda \xi_{n}^{2} \bar{\lambda}\right) t}\right)\right] \\
& +\left(\frac{F_{2 n}}{F_{1 n}}-\frac{F_{3 n}}{F_{1 n}} \frac{1}{1+\bar{\eta}}\right) \frac{\bar{\lambda}}{\lambda \xi_{n}^{2}}\left(1-\mathrm{e}^{-\left(\lambda \xi_{n}^{2} \bar{\lambda}\right) t}\right),
\end{aligned}
$$




$$
B_{4}(t)=\frac{2 \beta_{1}}{\lambda}\left(\mathrm{e}^{\lambda t / 2}-1\right)+\frac{\beta_{2}}{\lambda}\left(1-\mathrm{e}^{-\lambda t}\right) .
$$

\section{3 気渗透的理论计算与分析}

参照文献 [2], 取 $D_{0 \mathrm{~T}}=2.49 \times 10^{-7} \mathrm{~m}^{2} \mathrm{~s}^{-1}, \quad Q_{\mathrm{D}}=$ $5.115 \times 10^{4} \mathrm{~J} / \mathrm{mol}, \quad S_{0 \mathrm{~T}}=1.012 \mathrm{~mol} /\left(\mathrm{m}^{3} \sqrt{\mathrm{MPa}}\right)$ 和 $Q_{\mathrm{S}}=$ $1.014 \times 10^{4} \mathrm{~J} / \mathrm{mol}$, 并取 $T=300 \mathrm{~K}$ 进行解析理论计算, 分析気的渗透随有关物理和结构参数的变化规律. 本文以下将容器外気渗透总量 $Q_{\mathrm{p} 0}$ 与初始时刻容器 中的気量 $Q_{\mathrm{s}}$ 之比称为氞渗透比, 用 $\bar{Q}_{\mathrm{p} 0}$ 表示, 即 $\bar{Q}_{\mathrm{p} 0}=Q_{\mathrm{p} 0} / Q_{\mathrm{s}}$, 将気渗透总量衰变后的瞬时剩余量 $Q_{\mathrm{p} 1}$ 与初始时刻容器中的気量 $Q_{\mathrm{s}}$ 之比称为気渗透余 量比, 用 $\bar{Q}_{\mathrm{p} 1}$ 表示, 即 $\bar{Q}_{\mathrm{p} 1}=Q_{\mathrm{p} 1} / Q_{\mathrm{s}}$.

\section{1 容器外気渗透比和気渗透余量比随时间的变 化}

用 $\vartheta=\bar{r}_{\mathrm{o}} / \bar{r}_{\mathrm{i}}$ 表示容器径比, 当 $\vartheta=1.2$ 时, 针对 外表面氞浓度保持为零 $(\bar{\eta}=\infty)$ 和随気扩散而变化 $(\bar{\eta}=1)$ 两种情况, 图 1 给出了一容器気渗透比 $\bar{Q}_{\mathrm{p} 0}$ 和 気渗透余量比 $\bar{Q}_{\mathrm{p} 1}$ 随时间的变化曲线. 为了直观理解, 这里的时间变量直接是物理时间.

由图 1 可见, $\bar{\eta}=1$ 和 $\bar{\eta}=\infty$ 对应的 $\bar{Q}_{\mathrm{p} 0}$ 的相对差 别随时间延长而不断增大, 在两个半衰期内最大达 到 $65.3 \%$. 说明尽管 $\bar{Q}_{\mathrm{p} 0}$ 本身是微量的, $\bar{\eta}$ 的变化引 起 $\bar{Q}_{\mathrm{p} 0}$ 的绝对变化很小, 但就相对变化来说, 其影响 还是明显的. 对于 $\bar{\eta}=\infty$ 的情况, 在储存初期的一个 月内, $\bar{Q}_{\mathrm{p} 0}$ 与 $\bar{Q}_{\mathrm{p} 1}$ 随时间的变化很快, 且二者的差别 很小, 但此后随着时间延长, 二者的差别越来越大, 并在储存 193 个月时, 出现 $\bar{Q}_{\mathrm{p} 0}$ 继续增大, 而 $\bar{Q}_{\mathrm{p} 1}$ 减小 的情况, 也就是出现了容器外気衰变余量累积过大 而导致衰变速率大于渗透速率的情况. 这一点是很 好理解的, 因为容器外気量增大到一定程度时, 其因 衰变的减少量就会大于因渗透的增加量, 从而気的 剩余量就会减少. 对于 $\bar{\eta}=1$ 的情况, 从储存开始, $\bar{Q}_{\mathrm{p} 0}$ 与 $\bar{Q}_{\mathrm{p} 1}$ 都随时间缓慢变化, 二者的差别也是随时 间延长而越来越大, 在储存 217 个月时出现 $\bar{Q}_{\mathrm{p} 0}$ 继续
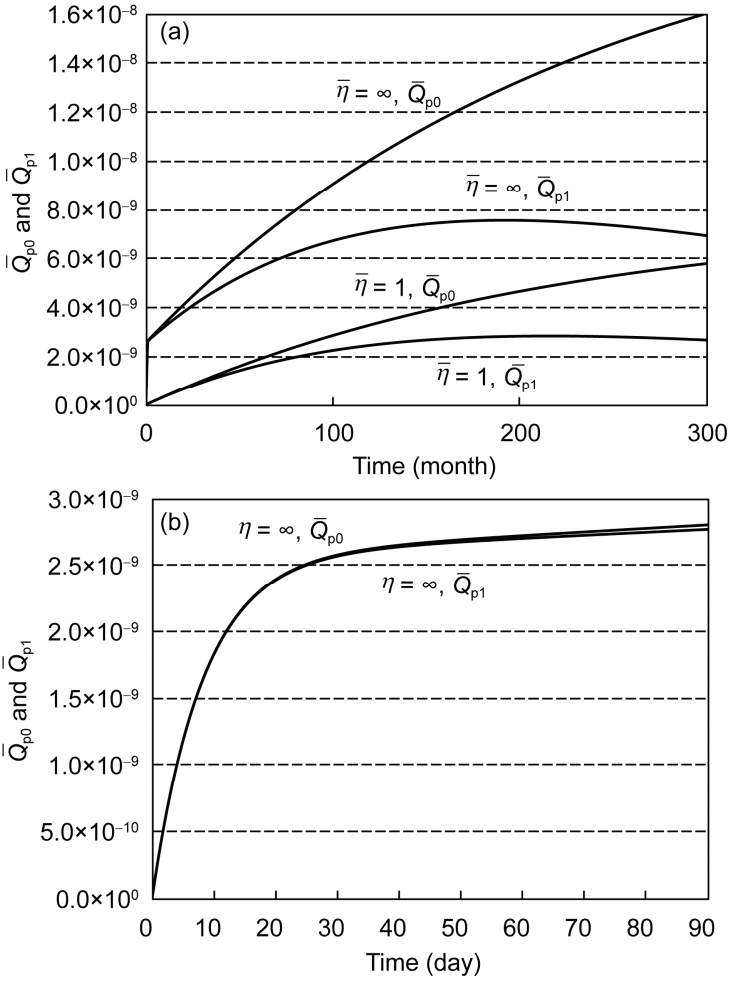

图 1 不同 $\bar{\eta}$ 情况下容器外気渗透比 $\bar{Q}_{\mathrm{p} 0}$ 和渗透余量比 $\bar{Q}_{\mathrm{p} 1}$ 随时间的变化

(a) 两个半衰期内的整体曲线; (b) 前 3 个月内的曲线((a)的局部放 大图)

增大、而 $\bar{Q}_{\mathrm{p} 1}$ 减小的情况. 这里 $\bar{Q}_{\mathrm{p} 1}-t$ 曲线说明, 在储 存过程中容器外部気渗透余量比存在一个最大值, 其出现的特征时间 $t$ 随 $\bar{\eta}$ 增大而略有延长.

\section{2 気渗透比 $\bar{Q}_{\mathrm{p} 0}$ 随对流传质系数 $\bar{\eta}$ 的变化规律}

同样取 $\vartheta=1.2$, 图 2 给出了储存半个和一个半 衰期时容器外気渗透比 $\bar{Q}_{\mathrm{p} 0}$ 的对数 $\log \bar{Q}_{\mathrm{p} 0}$ 随对流传 质系数的对数 $\log \bar{\eta}$ 的变化曲线. 图 2 中 $\bar{\eta}=0$ 表示容 器外表面处氚浓度梯度为零, 无気透过; $\bar{\eta}=\infty$ 表示 容器外表面気浓度为恒定的初始值, 氞浓度梯度最 大, 気透过量最大. 尽管在扩散过程控制渗透的情况 下, 实践中 $\bar{\eta}=0$ 和 $\bar{\eta}=\infty$ 两种极端的对流传质边界 条件都无法实现, 但从理论上讨论和认识 $\bar{\eta}$ 从零变 到无穷大时对気渗透的影响规律, 作为一种设计目 标, 对涉気材料的选用及研制、储気容器外环境的设 计还是有理论意义的. 因此图 2 给出了 $\bar{\eta}$ 从零变到无 


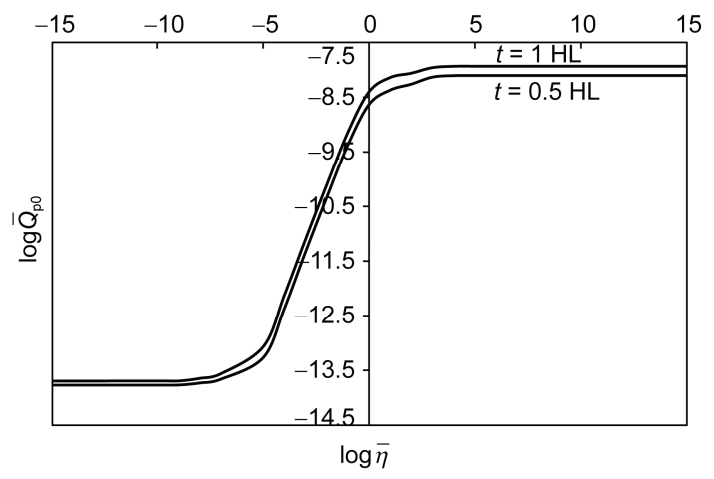

图 2 不同储存期限时 $\bar{Q}_{\mathrm{p} 0}$ 随 $\bar{\eta}$ 的变化

穷大时的结果.

由图 2 可见, 不同储存时间的 $\log \bar{Q}_{\mathrm{p} 0}-\log \bar{\eta}$ 曲线 是相似的, 储存时间长的曲线在储存时间短的曲线 的上方. 然后可见, $\log \bar{Q}_{\mathrm{p} 0}$ 随 $\log \bar{\eta}$ 的变化可分为 5 段, 第 1 段从 $\bar{\eta}=0$ 到约 $\bar{\eta}=10^{-7}$, 曲线几乎平直, $\bar{Q}_{\mathrm{p} 0}$ 随 $\bar{\eta}$ 的变化很小; 第 2 段从约 $\bar{\eta}=10^{-7}$ 到约 $\bar{\eta}=10^{-4}$, 曲线呈上凹型, $\bar{Q}_{\mathrm{p} 0}$ 随 $\bar{\eta}$ 的变化越来越快; 第 3 段从 约 $\bar{\eta}=10^{-4}$ 到约 $\bar{\eta}=10^{\circ}$, 曲线近似为斜率为 1 的斜直 线, 即 $\log \bar{Q}_{\mathrm{p} 0}$ 随 $\log \bar{\eta}$ 几乎线性增大; 第 4 段从约 $\bar{\eta}=10^{0}$ 到约 $\bar{\eta}=10^{4}$, 曲线呈上凸型, $\bar{Q}_{\mathrm{p} 0}$ 随 $\bar{\eta}$ 的变 化越来越慢; 第 5 段从 $\bar{\eta}=10^{4}$ 到 $\bar{\eta}=\infty$, 曲线又几乎 平直, $\bar{Q}_{\mathrm{p} 0}$ 随 $\bar{\eta}$ 的变化越来越小, 直至不变. 最后可 见, 在容器外表面浓度 $\left.c_{\mathrm{T}}\right|_{r=R_{\mathrm{o}}}=0$ 和 $\partial c_{\mathrm{T}} /\left.\partial r\right|_{r=R_{\mathrm{o}}}=0$ 的 两种极端边界条件下, $\bar{Q}_{\mathrm{p} 0}$ 的差别达到近 6 个数量级. 这些结果说明了如下 3 点. 一是在 $\bar{\eta}$ 小于某一值或大 于某一值后, $\bar{Q}_{\mathrm{p} 0}$ 随 $\bar{\eta}$ 的变化可以忽略. 当然, $\bar{\eta}$ 小 于某一值意味着 $\bar{\eta}$ 趋近于 0 , 即第三类边界条件退化 为第二类边界条件, 容器外表面几乎无気渗透, 这样 的情况在扩散过程控制渗透的情况下事实上难于达 到, 因为当 $\bar{\eta}$ 太小时意味着扩散很慢, 这样对渗透的 控制就自然由扩散过程转化为表面过程了. 二是存 在一个 $\bar{\eta}$ 的范围, 在该范围内 $\log \bar{Q}_{\mathrm{p} 0}$ 随 $\log \bar{\eta}$ 近似线 性变化. 三是就相对值来说, $\bar{\eta}$ 对 $\bar{Q}_{\mathrm{p} 0}$ 的影响是明显 的, 若实际的对流传质边界条件与两种极端条件 $\left.c_{\mathrm{T}}\right|_{r=R_{\mathrm{o}}}=0$ 或 $\partial c_{\mathrm{T}} /\left.\partial r\right|_{r=R_{\mathrm{o}}}=0$ 相差太大, 则应该采用文 献[11]中的第三类边界条件进行建模和求解器壁中
的気浓度以及渗透量, 以得到准确的结果. 这一点也 正是本节分析要说明的一个问题.

\section{3 気渗透比 $\bar{Q}_{\mathrm{p} 0}$ 和余量比 $\bar{Q}_{\mathrm{p} 1}$ 随容器径比 $\vartheta$ 的变 化规律}

为了在确保容器强度的同时也尽量利用容器壁 厚来减小気渗透量, 有必要了解気渗透量随径比的 变化规律. 图 3 针对 $\bar{\eta}=1$ 和储存一个半衰期的情况, 给出了気渗透比 $\bar{Q}_{\mathrm{p} 0}$ 和気余量比 $\bar{Q}_{\mathrm{p} 1}$ 随容器径比 $\vartheta$ 的 变化曲线. 可见, $\bar{Q}_{\mathrm{p} 0}-\vartheta$ 曲线和 $\bar{Q}_{\mathrm{p} 1}-\vartheta$ 曲线也是相 似的, 都呈单调减小特征, 即随着 $\vartheta$ 增大, 氞渗透比 减小; 曲线上存在一个 $\vartheta \approx 1.046$ 的特征值点, 当径 比小于该特征值时, $\bar{Q}_{\mathrm{p} 0}$ 和 $\bar{Q}_{\mathrm{p} 1}$ 随 $\vartheta$ 的增大而迅速减 小, 当径比大于该特征值时, $\bar{Q}_{\mathrm{p} 0}$ 和 $\bar{Q}_{\mathrm{p} 1}$ 随 $\vartheta$ 的增大 而减小得非常缓慢. 这一结果说明, 单就気渗透而言, 必须使容器的径比大于该特征值, 以提高容器壁的 抗気渗透性能. 当然, 由于容器强度和抗気渗透性能 都随径比增大而增强, 因此由于实际材料力学性能 的限制, 在现有的高压储気容器设计中, 从强度角度 设计的径比一般都比该特征值大很多.

\section{4 实验结果与分析}

本文研究的储気高压容器被置放在一个封闭的 外包装容器中, 二者之间的空腔(本文称为外空腔)中 充适量氩保护气, 从容器外表面渗透出来的気及其 衰变氦-3 主要就存留在该外空腔中.

由于缺乏容器外表面对流传质系数 $\bar{\eta}$ 的确切数 据, 且该数据也确实难以测试, 因此这里首先讨论一

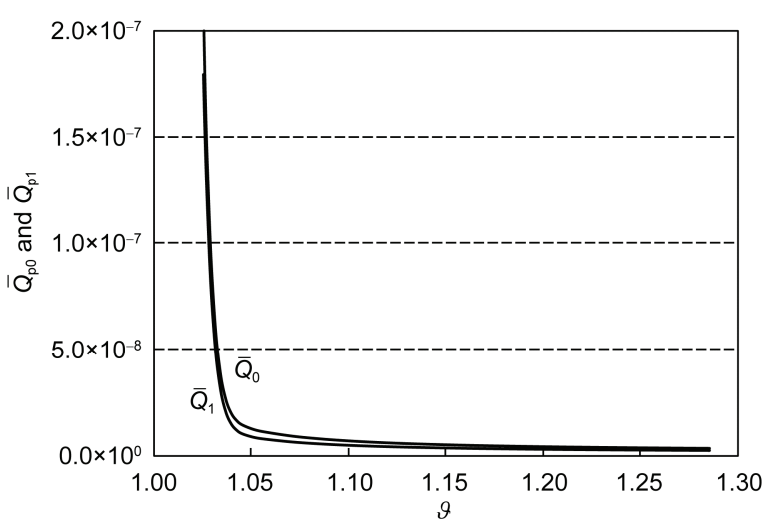

图 3 储存一个半衰期时 $\bar{Q}_{\mathrm{p} 0}$ 与 $\bar{Q}_{\mathrm{p} 1}$ 随径比的变化 
个确定 $\bar{\eta}$ 值的方法. 根据文献[2], 基于 Sieverts 定律 的(2)式也适用于容器外表面, 因此在求得外表面浓 度后, 由(2)式就可反求出外空腔中気的压强, 记为 $P_{\mathrm{T}}^{\prime}$. 同时在求得渗透过容器外表面的気的衰变剩余 量 $Q_{\mathrm{p} 1}$ 后, 也可求出外空腔中与 $P_{\mathrm{T}}^{\prime}$ 相应的気的压强, 记为 $P_{\mathrm{T}}^{\prime \prime}$. 显然求解 $P_{\mathrm{T}}^{\prime}$ 不需利用外空腔的体积, 而求 解 $P_{\mathrm{T}}^{\prime \prime}$ 要利用外空腔的体积, 因此两者的求解是独立 的. 当 $\bar{\eta}$ 值与实际相符时, 应有 $P_{\mathrm{T}}^{\prime}=P_{\mathrm{T}}^{\prime \prime}$. 这样, 通过 不断调整 $\bar{\eta}$ 值, 直至 $P_{\mathrm{T}}^{\prime}=P_{\mathrm{T}}^{\prime \prime}$ 时, 所调整到的 $\bar{\eta}$ 值就 是实际情况下的值. 调整过程 $P_{\mathrm{T}}^{\prime}$ 与 $P_{\mathrm{T}}^{\prime \prime}$ 的对比情况随 $\bar{\eta}$ 的变化及最终得到的 $\bar{\eta}$ 值见表 1 . 由表 1 中数据可 见, 只要画出 $P_{\mathrm{T}}^{\prime}$ 与 $P_{\mathrm{T}}^{\prime \prime}$ 随 $\bar{\eta}$ 的变化曲线, 两条曲线的 交点对应的 $\bar{\eta}$ 值就是所求的实际 $\bar{\eta}$ 值. 本文算例中 实际 $\bar{\eta}$ 值为 1705 . 该方法实际上是同时利用 Sieverts 定律和第三类传质边界条件求解固-气对流传质系数 的一种新方法, 它克服了难于准确实测 $\bar{\eta}$ 值的困难, 特别适用于固溶气体原子在表面复合后向封闭外空 腔内传递的情况.

采用球形高压容器进行较长时期的気储存实验 和测试, 容器的材料和几何尺寸与第三部分理论计 算中的相同, 其径比 $\vartheta=1.2$, 内部初始気容量也相 同. 测试时, 在不同时间对外空腔的氩保护气取样, 采用正比计数气相気测量方法测量样品中的気浓度, 再换算为気原子摩尔数, 最后除以初始时刻容器中
気的总摩尔数 $Q_{\mathrm{s}}$, 得到任意时刻容器外部总気量的 衰变剩余量比 $\bar{Q}_{1}$, 如表 2 . 这里为了客观反映实测情 况, 表中实测原始值没有进行误差校正, 但为了与计 算值进行比较, 表 2 中也给出了按图 1 中相应曲线的 上凸特征对 6 个实测原始数据进行 Excel 趋势线拟合 而得出的数据, 即表中的特征线拟合值.

表 2 中同时给出了取 $\bar{\eta}=1705$ 和 $\bar{\eta}=\infty$ (相当于 外表面気浓度保持为零) 时本文的理论计算值 $\bar{Q}_{\mathrm{p} 1}$ 和 采用稳态渗透理论公式 $Q_{\mathrm{p} 0}=4 \pi R_{\mathrm{o}}^{2} D_{\mathrm{T}} S_{\mathrm{T}} \sqrt{P_{\mathrm{T} 0}} t / h$ 对应 的衰变剩余量公式 $Q_{\mathrm{p} 1}=4 \pi R_{\mathrm{o}}^{2} D_{\mathrm{T}} S_{\mathrm{T}} \sqrt{P_{\mathrm{T} 0}}\left(1-\mathrm{e}^{-\lambda t}\right) /$ ( $\lambda h$ ) 计算的理论值 $\bar{Q}_{\mathrm{p} 1}$.

由表 2 中数据可见, 稳态理论计算值比瞬态理论 计算值大两个多数量级, 说明对于长期储気的渗透 问题，采用稳态理论计算会导致过大的误差. 而瞬态 理论计算值总体上与实测值处在同一数量级. 当然, 由于取样操作过程中気的流失、氩保护气中氮向外包 装容器中的渗透等、以及気的自身衰变减少, 实测值 略小于瞬态理论计算值. 另外, 从实测原始值随时间 的变化没有单调增加的规律也可看到, 实测值本身 确实存在一定误差, 因为由图 1 可知渗透量及其衰变 后剩余量在几个半衰期内应该单调增加. 尽管如此, 总的来说, 对于如此微量的气氛, 实测和瞬态理论计 算能够如此接近，已足以相互佐证彼此的合理性.

实验和理论结果都表明, 由于采用了特种抗氢

表 1 调整 $\bar{\eta}$ 得到的 $\boldsymbol{P}_{\mathrm{T}}^{\prime}$ 与 $\boldsymbol{P}_{\mathrm{T}}^{\prime \prime}$

\begin{tabular}{cccccc}
\hline $\bar{\eta}$ & 1600 & 1700 & 1705 & 1720 & 1750 \\
\hline$P_{\mathrm{T}}^{\prime}(\mathrm{Pa})$ & $1.8422 \times 10^{-4}$ & $1.6140 \times 10^{-4}$ & $1.5980 \times 10^{-4}$ & $1.5744 \times 10^{-4}$ \\
$P_{\mathrm{T}}^{\prime \prime}(\mathrm{Pa})$ & $1.5936 \times 10^{-4}$ & $1.5975 \times 10^{-4}$ & $1.5980 \times 10^{-4}$ & $1.5985 \times 10^{-4}$ & $1.5997 \times 10^{-4}$ \\
\hline
\end{tabular}

表 2 渗透気的衰变余量比 $\bar{Q}_{1}$

\begin{tabular}{|c|c|c|c|c|c|}
\hline \multirow{2}{*}{ 储存年限 (年) } & \multicolumn{2}{|c|}{ 实测值 $\bar{Q}_{1}$} & \multicolumn{2}{|c|}{ 瞬态理论计算值 $\bar{Q}_{\mathrm{p} 1}$} & \multirow{2}{*}{$\frac{\text { 稳态理论计算值 } \bar{Q}_{\mathrm{p} 1}}{\bar{\eta}=\infty}$} \\
\hline & 原始值 & 特征线拟合值 & $\bar{\eta}=1705$ & $\bar{\eta}=\infty$ & \\
\hline 6 & $1.3153 \times 10^{-9}$ & $1.3186 \times 10^{-9}$ & $4.0734 \times 10^{-9}$ & $6.011 \times 10^{-9}$ & $7.006 \times 10^{-7}$ \\
\hline 12 & $1.9444 \times 10^{-9}$ & $1.9023 \times 10^{-9}$ & $4.9878 \times 10^{-9}$ & $7.358 \times 10^{-9}$ & $1.201 \times 10^{-6}$ \\
\hline 13 & $6.2906 \times 10^{-10}$ & $1.9697 \times 10^{-9}$ & $5.0485 \times 10^{-9}$ & $7.451 \times 10^{-9}$ & $1.269 \times 10^{-6}$ \\
\hline 13.5 & $3.6028 \times 10^{-9}$ & $2.0015 \times 10^{-9}$ & $5.0712 \times 10^{-9}$ & $7.485 \times 10^{-9}$ & $1.301 \times 10^{-6}$ \\
\hline 14 & $2.0130 \times 10^{-9}$ & $2.0321 \times 10^{-9}$ & $5.0892 \times 10^{-9}$ & $7.510 \times 10^{-9}$ & $1.333 \times 10^{-6}$ \\
\hline
\end{tabular}


钢制造储気容器, 在较长的储存期内, 所研究实例容 器外部気的渗透总量及衰变后剩余量比内容気的初 始总量约小 8 个数量级, 这说明渗透导致的容器内部 気流失可以忽略. 但由于気被吸入人体后会导致严 重的内照射危害, 而当氞的初始总量较大时, 其渗透 量的衰变后剩余量也会随之增大, 因此渗透気对容 器外部环境的污染和对人员的危害仍是需要关注的.

\section{5 结论}

1) 针对容器外表面为一般对流传质边界条件的 情况, 从近年最新的気浓度公式出发, 导出了长期储 氞高压容器中氞的渗透量和衰变后剩余量公式.

2) 解析计算给出了容器外気渗透总量和衰变后 剩余量随储存时间的变化, 结果表明気渗透总量随 时间延长而单调增加, 剩余量随时间延长表现为先 增加后减小的规律; 给出了気渗透总量随外表面对 流传质系数的变化, 指出对流传质系数在 $0 \sim \infty$ 内存
在 4 个特征值点, 各特征值点前、后気渗透总量随对 流传质系数的变化规律不同; 给出了気渗透总量和 剩余量随容器壁厚的变化, 结果表明存在一个壁厚 的特征值, 在其前, 気渗透总量和剩余量随壁厚变化 很快, 在其后, 变化很慢. 这些规律及特征不仅是对 自然规律的新揭示, 而且可以指导储気容器的设计 和储存管理方式的改进.

3) 实验测试给出了気渗透量的衰变后剩余量数 据, 比较表明, 总体来说实测结果与瞬态渗透理论计 算结果吻合, 但采用稳态渗透理论的计算结果则有 过大的误差.

4) 实验和理论结果都表明, 由于采用了特种抗 氢钢, 在较长储存期内, 所研究实例容器外部気的总 量比内容気总量约小 8 个数量级, 渗透导致的容器内 部気流失可以忽略, 只需关注容器外部环境中的気 污染.

5) 提出了同时利用 Sieverts 定律和第三类对流 传质边界条件求解固-气对流传质系数的一种新方法.

\section{参考文献}

1 但贵萍, 杜阳, 杨勇, 等. 気污染气氛净化及关键技术研究. 全国核与辐射设施退役学术研讨会论文集. 北京: 中国核学会, 2007. 378-385

2 王佩璇, 宋家树. 材料中的氦及気渗透. 北京: 国防工业出版社, 2002. 53-81

3 Shiraishi T, Nishikawa M, Tamaguchi T, et al. Permeation of multi-component hydrogen isotopes through austenitic stainless steels. J Nucl Mater, 1999, 273: 60-65

4 姚振宇, 郝嘉琨, 周长善, 等. 复合膜对 316L 不锈钢気渗透性能的影响. 原子能科学技术, 2000, 34: 65-70

5 Marchi C S, Somerday B, Robinson S. Permeability, solubility and diffusivity of hydrogen isotopes in stainless steels at high gas pressures. WSRC-STI-2007-00579

6 王进军, 刘占旗, 战景明. $\mathrm{Al}_{2} \mathrm{O}_{3}(\mathrm{SiO} x) / \mathrm{PVC}$ 复合材料的研制和防氞性能测试. 辐射防护, 2009, 29: 300-304

7 杜良, 但贵萍, 曾俊辉. SiC 薄膜的阻気性能研究. 核技术, 2009, 32: 23-26

8 宋勇, 黄群英, 汪卫华, 等. ITER 中国液态锂铅实验包层模块気渗透分析. 核科学与工程, 2006, 26: 188-192

9 谢波, 翁葵平. 锂铅合金中気扩散行为的理论研究. 分子科学学报, 2009, 25: 352-356

10 Liu Y D, Yin Y H, Tan Y, et al. Research into tritium and helium-3 contents distributions in steel wall of spherical pressure vessel. Sci China Tech Sci, 2011, 54: 1521-1524

11 刘远东, 尹益辉, 谭云. 一般边界条件下球形压力容器钢壁中気和氦-3 的浓度变化规律研究. 物理学报, 2012, 61: 156601 


\title{
Analysis on tritium permeation in spherical pressure vessel
}

\author{
YIN YiHui ${ }^{1}$, LIU YuanDong ${ }^{1}$, CHEN ChangAn ${ }^{2} \&$ TAN Yun $^{1}$ \\ ${ }^{1}$ Institute of Systems Engineering, China Academy of Engineering Physics, Mianyang 621900; \\ ${ }^{2}$ National Defense Science and Technology Laboratory on Surface Physics and Chemistry, Mianyang 621907
}

\begin{abstract}
Most of the tritium quantity stored in a pressure vessel decays into helium-3 and a tiny part permeates the inner surface of the vessel and enters the wall material with time. The tritium in the wall material not only diffuses in the wall and till permeates out of the vessel through the outer surface, but also decays into helium-3 which remains in the wall. The tritium permeating out of the vessel can contaminate environment and harm human being's health and safety, therefore it is necessary to realize and control the permeation quantity. Directing at this situation that outer surface of the vessel is provided with general mass transfer boundary condition and the tritium inside the vessel is van der Waals gas, and taking simultaneously both decay and permeation of tritium within the vessel and decay and diffusion and permeation of tritium having permeated into the wall material into consideration, the theoretical model was developed and the theoretical formulas of total permeation quantity and surplus after decay of tritium were deduced. Through analytical calculation, the curves of total permeation quantity and surplus after decay of tritium versus time, total permeation quantity of tritium versus both convective mass transfer coefficient of the outer surface and the wall thickness of the vessel were plotted and related laws were revealed. Corresponding to the theoretical calculation, a tritium stored experiment was performed, the sample of protecting Argon gas which was between the tritium stored vessel and an outer closed container was extracted, and the tritium content was measured by using the method of proportional counter. Then, through conversion, the transient surplus after decay of the tritium out of the vessel was obtained. The analytical calculations data were compared with experimented measurement ones, which indicates the reasonability of theoretical analysis. The obtained laws that tritium permeation quantity changes with such parameters as time etc. are useful for theoretical design of tritium storied vessels.
\end{abstract}

spherical pressure vessel, tritium decay, permeation, diffusion

doi: 10.1360/N092014-00364 